\title{
Discussion on the Effect of Introducing Clinical Pharmacists to Guide Clinical Medication in ICU
}

\author{
Dalai Wuyun
}

Pharmacy Department of Chifeng Municipal Hospital, Chifeng 024000, Neimeng, China. E-mail: wuyudl@163.com

\begin{abstract}
Objective: to investigate the effect of clinical pharmacists in ICU. Methods: 108 ICU patients from January 2018 to March 2020 were divided into the control group and the observation group, with 54 cases in each group. The control group used the previous medication route, while the observation group introduced clinical pharmacists to guide clinical medication. The infection rate and adverse reaction rate of the two groups were compared. Results: the infection rate of the observation group was $3.70 \%$, and that of the control group was $14.81 \%$, which was significantly lower than that of the control group $(\mathrm{P}<0.05)$. The adverse reaction rate of the observation group was 5.56\%, and that of the control group was $18.52 \%$, which was significantly lower than that of the control group $(\mathrm{P}<0.05)$. Conclusion: the introduction of clinical pharmacists to guide clinical medication in ICU can effectively control the infection, and reduce various adverse reactions during drug use, so as to realize the scientific and standardized use of drugs, and improve the efficiency and safety of drug use.
\end{abstract}

Keywords: ICU; Clinical Pharmacist; Guidance; Clinical Medication; Effect

\section{Introduction}

ICU is the abbreviation of intensive care unit, which mainly provides effective isolation and treatment places for severe and comatose patients to complete the rescue of patients. Generally speaking, ICU patients have a high degree of disease severity, and the disease changes quickly, so the treatment needs to be very timely and accurate $^{[1]}$. In the hospital, the clinical drug work is generally in the charge of the hospital pharmacists. Through the communication between pharmacists and doctors, nursing staff and management personnel, the latest drug information is provided for the treatment of patients, the consultation of rational drug use is completed, and the rationality of drug use is improved ${ }^{[2]}$. However, at present, Chinese pharmacists fail to give full play to their responsibilities, especially, the use of anti-infective drugs leads to the abuse of antibiotics, and increasing the inci- dence of nosocomial infection, which is more dangerous for ICU patients. Therefore, it is necessary to introduce clinical pharmacists to guide clinical medication and improve the rationality of drug use ${ }^{[3]}$. This paper analyzes and discusses the effect of clinical pharmacist's guidance in ICU.

\section{Data and methods}

\subsection{General information}

108 ICU patients from January 2018 to March 2020 were divided into control group and observation group, with 54 cases in each group. In the control group, the ratio of male to female was 28:26. The lower limit of age was 20 years; the upper limit was 76 years; and the median age was $(48.21 \pm 4.33)$ years. There were 14 cases in neurosurgery, 16 cases in general surgery, 18 cases in

Copyright (C) 2021 Dalai Wuyun

doi: 10.18686/aem.v10i1.186

This is an open-access article distributed under the terms of the Creative Commons Attribution Non-Commercial License

(http://creativecommons.org/licenses/by-nc/4.0/), which permits unrestricted non-commercial use, distribution, and reproduction in any medium, provided the original work is properly cited. 
thoracic surgery and 6 cases in orthopedics ${ }^{[4]}$. In the observation group, the ratio of male to female was 29:25. The lower limit of age was 19 years; the upper limit was 76 years; the median age was (47.86 \pm 4.59$)$ years. There were 15 cases in neurosurgery, 14 cases in general surgery, 19 cases in thoracic surgery and 6 cases in orthopedics. There was no significant difference in basic data between the two groups $(\mathrm{P}>0.05)$.

\subsection{Method}

In the control group, the previous drug management mode was still used. After obtaining the prescription, the drug was reviewed and distributed according to the prescription after it was confirmed to be correct.

The specific measures are as follows: (1) clinical pharmacists need to enter the ICU every day to make rounds with the attending doctors, often make acute inspections on patients and obtain the latest results of patients' examinations. It's necessary to timely judge the abnormality of disease treatment, and whether the patients have bad psychology, if so, it needs actively communicate, give pharmaceutical care, and take good medication, to ensure the standardization of clinical medication. (2) In general, the utilization rate of antibiotics is high in clinic, and the treatment of diseases is mainly anti infection. Pharmacists need to provide reasonable medication suggestions and monitoring points, and pay attention to the standardization of combined medication. If patients are older, they need to consider the age problem, pay attention to the indicators of kidney examination and adjust the drug dose in time, so as to pay attention to control the adverse reactions during the use of drugs, and report and analyze the adverse reactions in time. (3) We should pay attention to the guidance of drug use for nursing staff, and observe whether the administration method of nursing staff is correct. Although comatose patients have difficulty in swallowing, enteric coated drugs can’t be taken after crushing, so as to prevent the efficacy from being affected. In severe cases, toxic reactions will occur. Therefore, it is necessary to replace the drugs, so as to provide more targeted drug intervention measures for patients. (4) We should join in the procurement and evaluation of new drugs, establish a patient-centered drug intervention mode, and get in touch with the drug storehouse purchasing personnel to master the clinical feedback of new drugs, so as to make clear the drugs in the hospital, carry out clinical medication guidance with the patient as the center and better realize pharmaceutical care.

\subsection{Observation indexes}

First, the infection rates of the two groups were analyzed, including pulmonary infection, urinary system infection and respiratory tract infection.

Secondly, the adverse reaction rates of the two groups were analyzed, including diarrhea, nausea and vomiting and stress ulcer.

\subsection{Statistical analysis}

The data of each observation index were qualitative data; n (\%) was used to represent count data, $x^{2}$ test; $(\overline{\mathrm{X}} \pm \mathrm{s})$ was used to represent measurement data, and $\mathrm{t}$ means test. After statistical analysis by SPSS22.0 statistical soft package, if the difference was significant and $\mathrm{P}$ $<0.05$, it was statistically significant.

\section{Results}

\subsection{Analyzing the infection rate of the two groups}

The infection rate of the observation group was $3.70 \%$, and that of the control group was $14.81 \%$, which was significantly lower than that of the control group ( $\mathrm{P}$ $<0.05)$. See Table 1 for details.

Table 1. The infection rate of the two groups is analyzed

\begin{tabular}{|l|l|l|l|l|}
\hline Group & Pulmonary infection (n) & $\begin{array}{l}\text { Urinary tract infection } \\
(\mathbf{n})\end{array}$ & $\begin{array}{l}\text { Respiratory tract infec- } \\
\text { tion (n) }\end{array}$ & $\begin{array}{l}\text { Infection } \\
\text { rate (\%) }\end{array}$ \\
\hline Control group (n=54) & 2 & 3 & 3 & 14.81 \\
\hline Observation group (n=54) & 1 & 0 & 1 & 3.70 \\
\hline$x^{2}$ & -- & -- & -- & 3.967 \\
\hline$P$ & -- & -- & -- & 0.046 \\
\hline
\end{tabular}




\subsection{The adverse reaction rates of the two groups are analyzed}

The adverse reaction rate of the observation group was $5.56 \%$, and that of the control group was $18.52 \%$, which was significantly lower than that of the control group $(\mathrm{P}<0.05)$. See Table 2 for details.

Table 2. Analysis of adverse reaction rate of the two groups

\begin{tabular}{|l|l|l|l|l|}
\hline Group & Diarrhea (n) & Nausea and vomiting (n) & Stress ulcer (n) & Infection rate (\%) \\
\hline Control group (n=54) & 4 & 3 & 3 & 18.52 \\
\hline Observation group (n=54) & 1 & 1 & 1 & 5.56 \\
\hline$x^{2}$ & -- & -- & -- & 4.185 \\
\hline P & -- & -- & -- & 0.038 \\
\hline
\end{tabular}

\section{Discussion}

ICU patients are badly ill, so patients generally need to be combined with drugs. And because the immune system is damaged, leading to a high incidence of infection, so for patients, scientific and reasonable drug use is very important to ensure the safety of drug use ${ }^{[5]}$. Pharmacists can guide the interaction, contraindications and compatibility of drugs, to improve the rationality of drug use.

According to the data analysis in Table 1-2 above, the infection rate of the observation group was 3.70\%, and that of the control group was $14.81 \%$, which was significantly lower than that of the control group ( $\mathrm{P}<$ 0.05). The adverse reaction rate of the observation group was $5.56 \%$, and that of the control group was $18.52 \%$, which was significantly lower than that of the control group ( $\mathrm{P}<0.05)$. Analyzing the reasons: clinical pharmacists have rich pharmaceutical knowledge, and can design safer and more reasonable drug treatment plans with doctors ${ }^{[5]}$. Therefore, pharmacists participating in clinical work can help doctors determine the dosage of drugs and their adverse reactions, and help doctors solve the adverse reactions during drug treatment, so they can control the adverse reactions. After drug intervention, the use of antibiotics can be more standardized, and the treatment of drugs is more targeted, so the control effect of infection is more ideal ${ }^{[6]}$. Using drug intervention and controlling acid inhibitors at the same time can better adjust the gastric acid and control the incidence of stress ulcer, so drug guidance of clinical medication can give full play to its advantages. They can use their own basic knowledge of medicine, chemistry and drug analysis to choose more standardized drug regimen for patients, and which is conducive to the recovery of ICU patients ${ }^{[7]}$.

In conclusion, the introduction of clinical pharmacists to guide clinical medication in ICU can effectively control infection and reduce various adverse reactions during drug use, so as to achieve scientific and standardized use of drugs and improve drug use efficiency and safety.

\section{References}

1. Liao Y, Li F, Yan J, et al. The role of clinical pharmacists in the anti-infective treatment of ICU patients with septic shock. Chinese pharmacy 2020; 31(11): 1382-1386.

2. Chen X, Zhang J. Analysis of clinical pharmacists' intervention on 156 inpatient orders in ICU. Chinese pharmacy 2014; 25(22): 2031-2034.

3. Shi Y. Practice and experience of clinical pharmacists participating in pharmaceutical care for ICU patients with severe infection. Chinese pharmacy 2012; 23(26): 2494-2496.

4. Zhao N, Shi Y, Liu G, et al. Experience of clinical pharmacist participating in the treatment of one case of acute ivermectin poisoning. Journal of clinical drug therapy 2018; 16(9): 90-92.

5. Wang H, Zhang K, Liu N, et al. Identification and pharmaceutical intervention of potential drug interactions in ICU by clinical pharmacists. Chinese journal of hospital pharmacy 2018; 38(16): 17471749, 1758.

6. Liu Y, Che X, Gan L, et al. Construction and exploration of clinical pharmacist training mode of traditional Chinese medicine guided by critical care medicine department. Chinese journal of hospital pharmacy 2019; 39(13): 1317-1321.

7. Li J, Zhu D. Clinical pharmacists involved in a case of ectopic ACTH syndrome complicated with Aspergillus fumigatus infection. Journal of medical forum 2020; 41(6): 64-67. 